\title{
Sensitivity of Blocking Probability in the Generalized Engset Model for OBS
}

\author{
Jianan Zhang, Yu Peng, Eric W. M. Wong, Senior Member, IEEE, Moshe Zukerman, Fellow, IEEE
}

\begin{abstract}
The generalized Engset model can be applied to evaluate the blocking probability at an optical cross connect (OXC) of bufferless optical burst switching (OBS) system. For tractability, previous studies assumed that burst transmission time (on-time) and time intervals between bursts provided by the same input channel (off-time) are exponentially distributed. Here we aim to study the sensitivity of blocking probability to the shape of these distributions. Extensive numerical results demonstrate that the blocking probability is not very sensitive to on- and offtime distributions in general. We observe that higher variance of on- and off-time distributions may lead to better performance.
\end{abstract}

Index Terms-Blocking probability, optical burst switching (OBS), generalized Engset formula, traffic model, sensitivity.

\section{INTRODUCTION}

Optical burst switching (OBS) [1], [2] is a switching technology proposed for wavelength division multiplexing (WDM) networks. It intends to combine the benefits of optical circuit switching (OCS) and optical packet switching (OPS).

There are various versions of OBS, including OBS/JET [3] and OBS/JIT [4], where bursts contending for a group of wavelength channels at each optical cross connect (OXC) may not use a large number of input channels to justify Poisson arrivals. Thus, an OXC cannot be simply regarded as an $M / M / k / k$ queuing system. Moreover, the Engset model is inaccurate for loss based OBS system [5], [6]. Instead, the generalized Engset model [7] could be applied.

Although the model in [5], [8] and [9] gives exact blocking probability solutions at an $\mathrm{OXC}$ when, for each input wavelength, on- and off-times are exponentially distributed, it does not provide exact solutions for other distributions. For the Engset model, blocking is insensitive to the shape of these distributions. However, the generalized Engset model does not possess such a property. This raises the importance to investigate errors introduced by assuming exponential distribution to evaluate blocking probabilities for other distributions. In this letter, we evaluate such errors when on- and off-time distributions are deterministic, exponential, hypo-exponential, hyperexponential, Pareto and truncated Gaussian (to avoid negative

Manuscript received August 11, 2011. The associate editor coordinating the review of this letter and approving it for publication was J. Wang.

J. Zhang is an undergraduate student at the Electronic Engineering Department, Tsinghua University, Beijing, PRC. Y. Peng, E. W. M. Wong and M Zukerman are with the Electronic Engineering Department, City University of Hong Kong, Hong Kong SAR, PRC. The work on this paper was carried out while the first author was with the Electronic Engineering Department, City University of Hong Kong.

The work described in this paper was supported by a grant from the Research Grants Council of the Hong Kong Special Administrative Region, China [CityU 124709] and by a start-up grant from City University of Hong Kong [No. 9380044]. values). We observe that blocking probability is generally not very sensitive to the shape of distributions but traffic whose on- and off-time distributions have higher variance may have lower blocking probability.

\section{Methodology}

\section{A. Modeling of $O B S O X C$}

As in [5], we focus on a set of output wavelength channels in an output cable of an OXC. Suppose there are $F$ optical fibers in this cable, each of which carries $W$ wavelengths. Without wavelength conversion, an arriving burst on a given wavelength must use the same wavelength at the output, so only $F$ wavelength channels are available. With wavelength conversion, all $F W$ output channels are available for an arriving burst. These available wavelength channels are considered as $K$ servers. We use the term sources for the relevant input channels in each case. Without wavelength conversion, the sources are the input channels that have the same wavelength as the $F$ output channels. With wavelength conversion, the sources are all the relevant input channels that provide bursts to the $F W$ output channels. The number of sources is denoted by $M$. Each source transmits bursts as an on/off process, with mean on- and off-time equal to $1 / \mu$ and $1 / \lambda$, respectively.

If there is no output channel available for an arriving burst, the burst is dumped, in which case, it still occupies ("freezes") the input channel for the entire burst duration. We classify the sources to be free, busy and frozen. A source in its offtime is free and otherwise either busy, when its burst is being transmitted through an output channel, or frozen, when its burst is being dumped.

\section{B. Markovian models}

Cases where on- and off-times follow exponential, hyperexponential or hypo-exponential distributions lead themselves to exact Markov chain analyses. Dimensions of these models are listed in Table I. For brevity, we do not provide steady state equations here for all cases. Instead, we only present the case with hyper-exponentially distributed on-time and exponentially distributed off-time. See the extended version of this letter [10] for the steady state equations of all the Markovian models.

Consider a hyper-exponentially distributed random variable with the following probability density

$$
f(t)=p_{1} f_{\mu_{1}}(t)+p_{2} f_{\mu_{2}}(t) \quad\left(p_{1}+p_{2}=1\right)
$$

where $f_{\mu_{1}}(t)$ and $f_{\mu_{2}}(t)$ are probability densities of exponential distribution with parameters $\mu_{1}$ and $\mu_{2}$, respectively. Accordingly, for hyper-exponentially distributed on-time and 
TABLE I

MARKOVIAN MODELS

\begin{tabular}{|l|l|c|}
\hline On-time Distribution & Off-time Distribution & Dimensions \\
\hline Exponential & Exponential & 2 \\
Hyper-exponential & Exponential & 4 \\
Hypo-exponential & Exponential & 4 \\
Exponential & Hyper-exponential & 3 \\
Exponential & Hypo-exponential & 3 \\
Hyper-exponential & Hyper-exponential & 5 \\
Hypo-exponential & Hypo-exponential & 5 \\
\hline
\end{tabular}

TABLE II

NON-MARKOVIAN MODELS

\begin{tabular}{|l|l|}
\hline On-time Distribution & Off-time Distribution \\
\hline Deterministic & Exponential \\
Pareto & Exponential \\
Truncated Gaussian & Exponential \\
Exponential & Deterministic \\
Exponential & Pareto \\
Exponential & Truncated Gaussian \\
Deterministic & Deterministic \\
Pareto & Pareto \\
Truncated Gaussian & Truncated Gaussian \\
\hline
\end{tabular}

exponentially distributed off-time, we consider each busy or frozen source has two possible states. Sources transmitting bursts of exponentially distributed lengths with parameter $\mu_{1}$ and $\mu_{2}$ are said to be in states 1 and 2 , respectively. Let $\pi_{i, j, k, l}$ be the steady state probability that there are $i$ and $j$ busy sources in states 1 and 2 , respectively, and $k$ and $l$ frozen sources in states 1 and 2 , respectively $(0 \leq i+j \leq K$; $0 \leq k+l \leq M-K ; i, j, k, l \geq 0)$. For a free source, the rates to become busy or frozen in states 1 and 2 are $p \lambda$ and $(1-p) \lambda$, respectively. Then we have the following steady state equations. For $i+j<K$,

$$
\begin{aligned}
& {\left[(M-i-j-k-l) \lambda+(i+k) \mu_{1}+(j+l) \mu_{2}\right] \pi_{i, j, k, l}} \\
& \quad=(M-i+1-j-k-l) p \lambda \pi_{i-1, j, k, l} \\
& \quad+(M-i-j+1-k-l)(1-p) \lambda \pi_{i, j-1, k, l} \\
& \quad+(i+1) \mu_{1} \pi_{i+1, j, k, l}+(j+1) \mu_{2} \pi_{i, j+1, k, l} \\
& \quad+(k+1) \mu_{1} \pi_{i, j, k+1, l}+(l+1) \mu_{2} \pi_{i, j, k, l+1} .
\end{aligned}
$$

For $i+j=K$,

$$
\begin{aligned}
& {\left[(M-K-k-l) \lambda+(i+k) \mu_{1}+(j+l) \mu_{2}\right] \pi_{i, j, k, l}} \\
& \quad=(M-K+1-k-l) p \lambda\left(\pi_{i-1, j, k, l}+\pi_{i, j, k-1, l}\right) \\
& \quad+(M-K+1-k-l)(1-p) \lambda\left(\pi_{i, j-1, k, l}+\pi_{i, j, k, l-1}\right) \\
& \quad+(k+1) \mu_{1} \pi_{i, j, k+1, l}+(l+1) \mu_{2} \pi_{i, j, k, l+1} .
\end{aligned}
$$

Then we have the normalization equation:

$$
\sum_{i=0}^{K} \sum_{j=0}^{K-i M-K} \sum_{k=0}^{M-K-k} \sum_{l=0}^{M-k} \pi_{i, j, k, l}=1 .
$$

The offered load is given by

$T_{o}=\sum_{i=0}^{K} \sum_{j=0}^{K-i} \sum_{k=0}^{M-K} \sum_{l=0}^{M-K-k}(M-i-j-k-l) \lambda\left(\frac{p}{\mu_{1}}+\frac{1-p}{\mu_{2}}\right) \pi_{i, j, k, l}$.

The carried load is given by

$$
T_{c}=\sum_{i=0}^{K} \sum_{j=0}^{K-i M-K} \sum_{k=0}^{M} \sum_{l=0}^{M-K-k}\left(i \mu_{1}+j \mu_{2}\right)\left(\frac{p}{\mu_{1}}+\frac{1-p}{\mu_{2}}\right) \pi_{i, j, k, l} .
$$

The blocking probability is obtained by

$$
B=\frac{T_{o}-T_{c}}{T_{o}} \text {. }
$$

Although the computation time can be reduced by using matrix methods [11], for certain large size problems we rely on Markov chain simulations.

\section{Non-Markovian models}

When distributions of on- or off-time are deterministic, Pareto and truncated Gaussian, listed in Table II, the blocking probabilities are obtained by discrete event simulations.

\section{NUMERICAL RESULTS}

Aiming to investigate the errors introduced by assuming exponential on- and off-time distributions when evaluating blocking probabilities for the other distributions, we present here normalized histograms that estimate the error distributions. The depicted histograms are based on about 40,000 cases of calculations and simulations over a wide range of parameters.

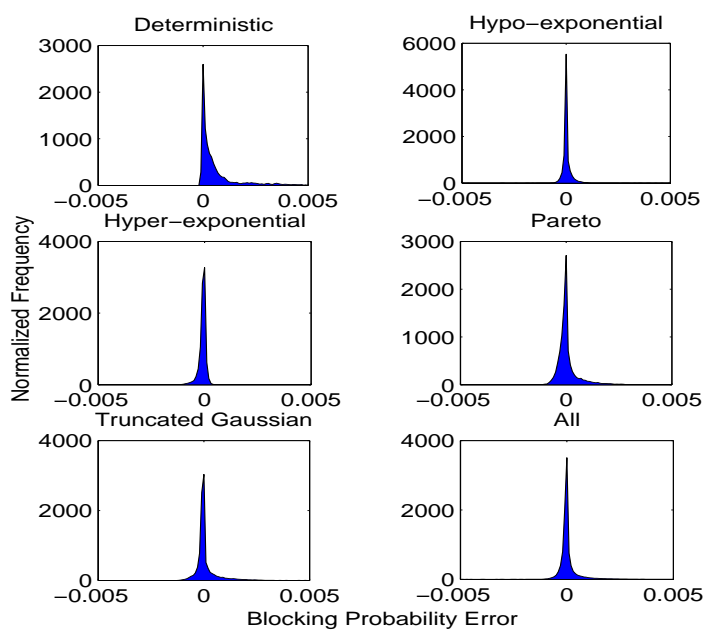

Fig. 1. Normalized histograms of blocking probability errors for cases with various on-time distributions and exponential off-time distribution.

In Fig. $1-3, \mu$ is fixed at $0.1 ; \lambda$ is randomly chosen between 0.01 and $10 ; M$ is selected based on a discrete uniform distribution among $3,4, \ldots 30$ and then $K$ is selected uniformly among $1,2, \ldots M-1$. In Fig. 1, we present error distribution histograms for cases where off-times follow exponential distribution and on-times follow the other distributions. In Fig. 2, we present error histograms for cases where on-times follow exponential distribution and off-times follow the other distributions. In Fig. 3, we present error histograms for cases where both on- and off-times follow the other distributions. They all demonstrate that the blocking probability is generally not very sensitive to the shape of on- and off-time distributions. However, it is more sensitive to the shape of off-time distributions compared with on-time distributions. As discussed below, there are cases that give larger blocking probability errors.

From the above figures we observe that when on- or offtime is deterministic, blocking probability is usually higher. 


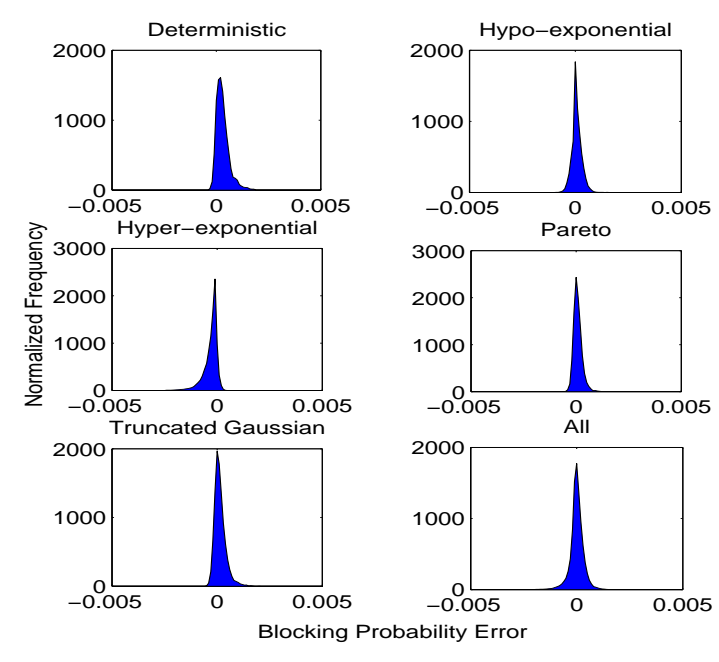

Fig. 2. Normalized histograms of blocking probability errors for cases with various off-time distributions and exponential on-time distribution.

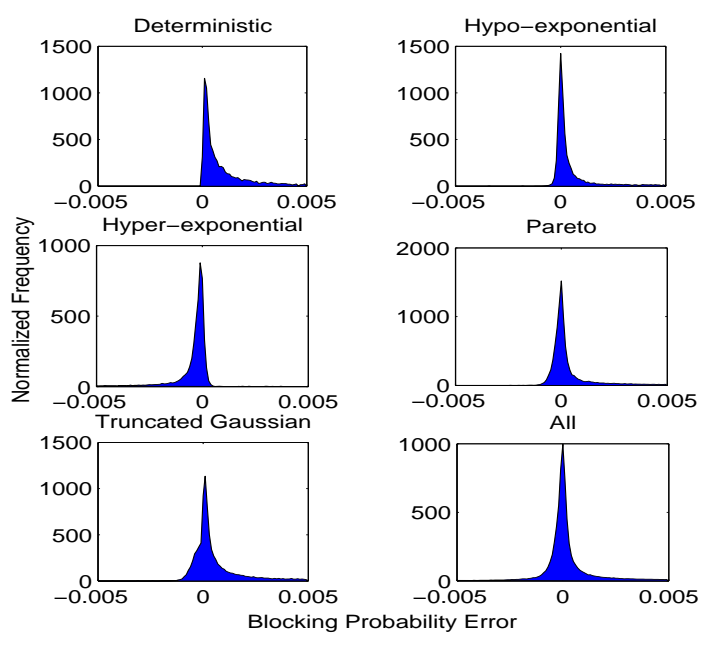

Fig. 3. Normalized histograms of blocking probability errors for cases with various on- and off-time distributions.

To explain this effect consider an example with two sources and one server. Assume that for each of the sources on-time and off-time are deterministic where the on-time is $\Delta+\varepsilon$ and the off-time is $\Delta-\varepsilon$ for arbitrarily small $\varepsilon$. In this case, all the bursts will collide, so arriving bursts will be dumped with probability of 0.5 , which is the highest possible blocking probability in a system of two identical sources and one server with the same mean on- and off-time. If we increase the variance of the off-time, the occurrences of longer off-time in one source allow bursts from the other source to access the server without collision, reducing the blocking probability. We have observed similar results in cases with larger values of $M$ and $K$ (see the extended version of this letter [10]).

Fig. 4 depicts blocking probability estimations for cases involving exponential on-time distribution and other off-time distributions. Blocking probability of the case where off-times follow exponential distribution was obtained by solving the steady state equations. The others were obtained by simulations. The 95\% confidence intervals based on Student-t distribution are smaller than plotted points and therefore not shown. Their radii are kept below $10^{-4}$. We observe that lower variance of the off-time distribution causes certain increase in blocking probability, so clearly, the insensitivity of the Engset model does not apply to the present case. Nevertheless, the variations in the blocking probability are small.

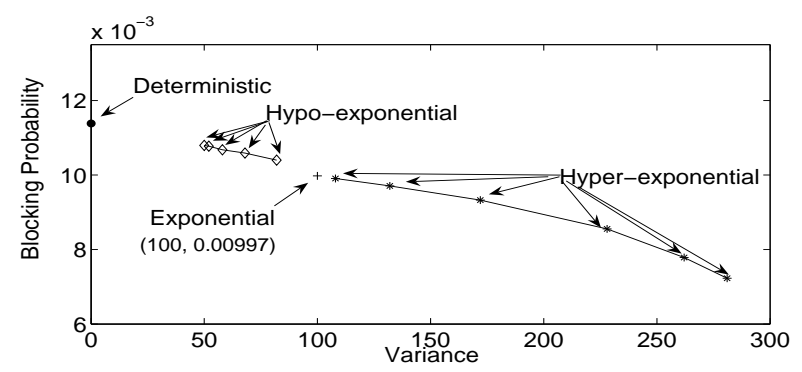

Fig. 4. Blocking probability vs. variance of off-time distribution with exponential on-time distribution for $M=100, K=75, \lambda=0.1, \mu=0.05$.

\section{CONCLUSION}

We have studied the sensitivity of blocking probability of bursts to the shape of on- and off-time distributions at an OBS OXC. Based on the tests studied, blocking probability is generally not very sensitive to the shape of the distributions of both on- and off-time, which justifies the use of the exponential distributions. Moreover, we have observed and explained the interesting phenomenon that lower variance may lead to higher blocking probability.

\section{REFERENCES}

[1] Y. Chen, C. Qiao, and X. Yu, "Optical burst switching: a new area in optical networking research," IEEE Network, vol. 18, no. 3, pp. $16-$ 23, May-Jun. 2004.

[2] Z. Rosberg, H. L. Vu, M. Zukerman, and J. White, "Performance analyses of optical burst switching networks," IEEE Journal on Selected Areas in Commuincations, vol. 21, no. 7, pp. 1187-1197, Sep. 2003.

[3] M. Yoo and C. Qiao, "Just-Enough-Time (JET): A high speed protocol for bursty traffic in optical networks," in IEEE/LEOS Technologies for a Global Information Infrastructure, 1997, pp. 79-90.

[4] J. Y. Wei, J. L. Pastor, R. S. Ramamurthy, and Y. Tsai, "Just-in-time optical burst switching for multiwavelength networks," in Broadband Communications, 1999, pp. 339-352.

[5] M. Zukerman, E. W. M. Wong, Z. Rosberg, G. M. Lee, and H. L. $\mathrm{Vu}$, "On teletraffic applications to OBS," IEEE Communications Letters, vol. 8, no. 2, pp. 116-118, Feb. 2004.

[6] E. W. M. Wong, A. Zalesky, and M. Zukerman, "A state-dependent approximation for the generalized Engset model," IEEE Communications Letters, vol. 13, no. 12, pp. 962-964, Dec. 2009.

[7] J. W. Cohen, “The generalized Engset formulae," Philips Telecommunication Review, vol. 18, pp. 158 - 170, 1957.

[8] A. Detti, V. Eramo, and M. Listanti, "Performance evaluation of a new technique for IP support in a WDM optical network: optical composite burst switching (OCBS)," Journal of Lightwave Technology, vol. 20, no. 2, pp. $154-165$, Feb. 2002.

[9] H. Overby, "Performance modelling of optical packet switched networks with the Engset traffic model," Optics Express, vol. 13, pp. 1685 - 1695, 2005.

[10] J. Zhang, Y. Peng, E. W. M. Wong, and M. Zukerman, "Sensitivity of blocking probability in the generalized Engset model for OBS (extended version)," Aug. 2011, Internal Technical Report. [Online]. Available: http://www.ee.cityu.edu.hk/ ewong/obs/sensitivity_full.pdf

[11] N. Akar and Y. Gunalay, "Stochastic analysis of finite population bufferless multiplexing in optical packet/burst switching systems." IEICE Transactions, pp. 342-345, 2007. 